\title{
A CONTRIBUTION TO THE TREATMENT OF PHYTOSIS OF . THE FEET *
}

\author{
MILLER B. HUTCHINS, M.D. \\ Atlanta, Ga.
}

As the term epidermophytosis seemed about to supplant the ancient titles (White, the author, and others), workers demonstrated that the tricophytons are the majority of the offenders, with others of the higher fungi (Wende-Collins) capable of producing disease. The term tinea, defined worm, was employed as far back as 1829 and has continued to cover several different dermatologic conditions. Tricophytosis, for years, and epidermophytosis, of late, bade fair to break the reign of tinea, but now we must coin a new name. Taenia means tapeworm; tinea, any worm.

While investigators seem not to have complied with Koch's law, the presence of these fungi, with resistance and recurrence of disease induced, as well as the usual sources and methods of infection, leaves little doubt of their pathogenicity. As to the distribution of this class of disease, it is at least universal, almost pandemic, among men; and for the simplest of reasons. Casual reference to "toe trouble" or "foot trouble" in conversation anywhere is more than likely to arouse interest, though it is a natural condition. Cases in children as young as 7 are in my records, an evidence of parental carelessness. I have never seen a very old person with the disease. There is a Y. M. C. A. building here, the shower baths of which are open to all members, whether resident or not. Even before the war, this disease was prevalent. During the war, lay over soldiers often used these baths. The disease has been so common of late that I have heard it referred to as the "M. A. disease." Public baths and showers, the more menacing, even golf club showers, are the leading sources of infection. Socks and stockings may actually bring the disease from the laundry, or reinfect the owner if, as Mitchell suggests, the organisms survive laundering. ${ }^{1}$ There is some evidence to support the belief that the

* A clinical paper, based on private cases.

1. Many housewives wash socks and stockings at home without boiling. Washerwomen do not boil them. The head of a large laundry states that these articles, of the same colors, are put in meshed bags and run through the mangle, with little heat, the cleansing chemical probably being sodium carbonate. They are then placed on hollow, metal forms, steam-heated to 180 or $200 \mathrm{~F}$., the drying process requiring from two and one-half to four minutes. I am now instructing patients to have hosiery boiled at home for at least fifteen minutes. 
fungi may be water-borne, as in a case supposedly contracted from a Georgia stream and another through a laceration of the bare sole of a young woman at a bathing beach near Detroit, though the latter infection could have come from a preceding infection of the foot. The family tub and towels, and the exchanging of footwear, provide other sources of infection.

The disease, while easily transinoculated by the patients' hands, or auto-inoculable, is very rarely directly contagious. A curious illustration of variations in personal local immunity is the frequency of involvement of one foot alone, this condition often persisting for months, even indefinitely. Such local persistence with frequent recurrences is also strong evidence of dormancy and periodic growth of retained organisms, rather than of positive reinfection in loco.

These fungi are known to have survived from five to nine months in a dry envelop, but the ultimate longevity of some of them is yet to be determined. They may rival the Egyptian wheat which germinated after reposing for 2,000 years in a tomb. Mitchell has shown viability of certain phyton forms after boiling in potassium hydroxid solution.

That the organisms may lie dormant during cool or cold weather, either in contaminated articles of apparel or actually in the epidermis, in spite of normal desquamation, is well established. Recurrence of disease in practically the same areas occupied during the preceding summer and the depth from which lesions may arise, as on the plantar surfaces, at least favors the probability of epidermal hibernation. Even in Atlanta, where we average less than one freeze a week in winter and the temperature may get up to $60 \mathrm{~F}$. or higher, many cases give no trouble but reappear as the average daytime temperature reaches $70 \mathrm{~F}$. I see very few of the phytoses in winter, but very many in summer. Yet there are cases even in Boston and Detroit that are unchecked by cold weather, and they are also seen in Atlanta.

Whether being in heated homes, cars and offices, with a minimum of exposure to cold, or whether the variety of organism is responsible for these exceptions, we have no positive knowledge. That warmth and moisture, not necessarily hyperhidrosis, do encourage growth of these fungi is known. In this connection, it is a curious fact that we rarely see phytic infection of the feet in cases of bromidroses.

The depth to which the phytons penetrate, as in plantar regions and the fourth interspace, as well as in callosities, constitutes the primary reason for persistent recurrences and difficulty of cure. Extracorporeal destruction of germs or fungi and destruction in vivo are vastly different propositions. Dry air and sunlight may kill tubercle bacilli, drying suppress gonococci, soap destroy Spirochetae pallidae and radiation disintegrate cancer cells, but for cure every organism or 
cell must yield. When contact is established, as in vitro, such accomplishment may be easy. In the tissues, we must get at the cause or depend on the natural resistant forces of the body cells, reinforced if possible. Schamberg's weak iodin destroys the fungi in vitro, and tincture of iodin is practically useless as applied by patients to these foot conditions. Ruggles' tincture of iodin 1, spirits of camphor 7, did well until his patient went on a six weeks' vacation and left off treatment, with the usual relapse.

Ripened or developed lesions yield rather readily to almost any good dermatologic treatment, most of which are more or less germicidal, though not by far so rapidly as a simple dermatitis. Hidden organisms delay recovery, just as they, with now and then a reinfection, prevent permanent cure. After getting relief, the majority of patients disappear.

\section{TREATMENT}

In order to effect a cure, it is imperative that we rid the patient of all harbored fungi, and, in doing this, endeavor to adapt remedies to the destruction of organisms present in articles worn. Whitfield's principles of treatment must be followed in one particular at least, and to extremes. These cases require constant desquamating and exfoliating; in other words, persistent and intensive peeling. This is best accomplished with salicylic acid, combinations of methods to be mentioned later activating its effect. Benzoic acid is probably almost inert as a fungicide, as shown by Schamberg.

Pus cases, secondary coccic infections, have needed but a single remedy to clear them up-in from twenty-four to forty-eight hours, whether there be a few pustules or the greater part of the plantar surface is undermined. Small lesions are broken up and the larger buttonholed in such manner as to prevent reclosure and yet leave sufficient epidermis for protection of tender surfaces until new can form. The official ointment of ammoniated mercury, in half or full strength, is specific. A few pus conditions may be too inflammatory, or, more likely, the lesions may have been stained with tincture of iodin, the latter condition contraindicating mercury. A mild boric ointment is satisfactory as a beginning in these cases.

Ointments are heavily applied, with cotton to retain them, and changed at least twice in twenty-four hours. Because of their messiness, doubtful utility and injury to footwear, I do not employ ointments in the nonpyodermic cases, save at night in conditions tending to become very dry.

Stains and dyes have been avoided because of their character as well as doubt of their ability to reach or affect the phytons. Treatment has been limited largely to powders and to mechanical procedures. 
It may be stated with positiveness that the majority of cases of phytosis must be treated after the war cry of the Tank Corps, very few yielding definite results under gentle management. Soap, water and scrubbing (Williams) must be employed, not only for cleanliness but also to get rid of as much dead epithelium and as many fungi as possible. Vesicles are broken up, bullae and large subepidermic lakes torn to pieces or buttonholed, as in the pus conditions, and shreds are cut or peeled away in all cases. Plintar callosities are cleansed with soap and water or gasoline and covered with salicylic acid, roofed over with narrow overlapping strips of adhesive plaster-wide sections do not fit-and this dressing renewed as often as it loosens. As the seminecrosed, broken-up, rubber-like epidermis begins to separate, it is removed. The salicylic acid may also be employed on interspace accumulations, particularly the wide fourth. At the same time, salicylic acid is used in all powders, $1: 8$, or stronger, often alone, on the feet and in socks.

The gamut has been run with everything from a little boric acid even through ground-up hexamethylenamin and, occasionally, thymol iodid or calomel, to sulphur. Salicylic acid in the socks produces definite exfoliation of thick epidermis, as of plantar surfaces, and desquamation alone of lateral and dorsal areas, partly through greater contact from drifting down in the former instance, but also because the thickened epidermis is farther from its blood supply, and so less vital. It not only opens their lair,' but possibly has some destructive action on the organisms; spores, as always, being perhaps the hardest to kill.

Proof that going into the treatment of the phytoses is entering a maze in which we wander rather vainly seeking a definite objective exists in the many remedies tried and abandoned or employed with scant success. .

If formaldehyd vapor from an open bottle in the laboratory "killed all the ringworm cultures," why could the liquid or vapor not act in phytosis? Vapor was used under a rubber cap, in scalp cases. The disease required its usual time for cure. There is no practical way of applying vapor to foot cases, and any percentage of the liquid is too irritating and of slight penetration.

Hexamethylenamin grossly pulverized was put in socks, shoes and slippers, with the hope of getting some free formaldehyd. The soles were tanned and thickened, but vesicles and small bullae continued to appear even in the leathery tissue. Leather insoles were ruined by the drug. Benzin, or gasoline, applied to perfect sheaths of little black bugs on new shoots of grapevines killed both the bugs and a foot or two of new vine. Applied to broken vesicular points on the toes, it intensely aggravated the itching. Saturating the socks with it in the hope of sterilizing the feet and foot coverings had no apparent effect. 
Surgical solutions were not considered worthy of trial and germicidal ointments, even the pus destructive ointment of ammoniated mercury, has been of doubtful utility in the phytoses. Any effect from the roentgen rays was simply due to cell reaction, and transitory. On several occasions, new erythematovesicular patches were treated by momentary freezing with ethyl chlorid, in the hope that refrigeration might hasten recovery. Carbon dioxid snow would have been cheaper, but the treatment was applied in the patient's room. Results of this brief freezing were uncertain.

In desperation, a physician even applied: Oleate of mercury and olive oil, four parts each, and sulphuric ether, sixty parts (mixed), an instantly effective remedy for pediculosis pubis and their ova. Even this mixture had practically no effect, except to ruin the shoes.

Powder prescriptions have always had talcum as the vehicle, since it is insoluble and keeps dry.

Powders have run from the ancient: salicylic acid and boric acid one part each, pulverized zinc oxid from four to eight parts, and pulverized talcum to make thirty parts (mixed); on through: salicylic acid, from two to four parts, benzoic acid, four parts, and pulverized talcum to make thirty parts (mixed); and salicylic acid, two parts, calomel, four parts, and pulverized talcum to make thirty parts (mixed).

Even pulverized alum has been added to some of these prescriptions for its drying effect; and, finally: salicylic acid and precipitated sulphur, fifteen parts each (mixed).

He rubbed as frequently as possible on the hands of a patient who could treat them but little during the day; applying at night: salicylic acid and precipitated sulphur, four parts of each, and zinc oxid ointment to make thirty parts (mixed), on all affected parts. The patient has done well.

A paste of sulphur and lard hastened recovery in one case. Whitfield's ointment, stronger than the original, is still employed in some cases at night, and occasionally, the ammoniated ointment or petrolatum.

It will be seen from the foregoing that salicylic acid is depended on as the sheet anchor in all prescriptions.

There has been no opportunity to evaluate results from soaking the feet at night in hot water, salt water or ice water, or from washing them with gasoline, the majority of patients having been unwilling to fight the disease to a finish.

In the light of later experience, and through study of old case records, I am inclined to believe that pompholyx is not a distinct disease, as we get these exact lesions, "sago-grains" and all, in the phytoses. 
CASE 1.-J. W. C., aged 23, first seen, May 25, 1892, had six weeks before developed deep bullae and some excoriations on the left toes and foot. When examined, there were several ulcers, with pus and blood, and anterior plantar, flat bullae and denudations. Much of the pus was green. "Antiseptic" salves and powders were employed, and some "mixed treatment" was given, on a dubious history. The foot was well in fifteen days.

November 2: Pompholyx which had developed in the sole of left foot as a group of deep vesicles was drying up.

April 6, 1893: The patient had another attack of pompholyx, with deepseated, shot-like vesicular lesions.

June 2: New vesicles developed. The patient had been using:

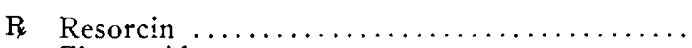

Zinc oxid $\ldots \ldots \ldots \ldots \ldots \ldots \ldots \ldots \ldots \ldots \ldots \ldots \ldots$

Pulverized talcum................ s. ad

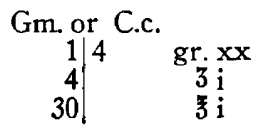

July 14: Bullae developed beneath the first and second interspaces of both feet. Asiatic pills were prescribed, and for local application:

R Resorcin (pulverized) $\ldots \ldots \ldots \ldots \ldots \ldots \ldots$

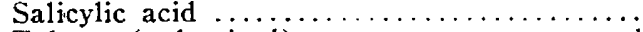

Talcum (pulverized) $\ldots \ldots \ldots \ldots \ldots \ldots \ldots$ ad

\begin{tabular}{r|ll} 
Gm. or C.c. & \\
1 & 4 & $\mathrm{gr} . \mathrm{xx}$ \\
1 & & $\mathrm{gr} . \times \mathrm{vv}$ \\
30 & & $\mathbf{3} \mathrm{j}$
\end{tabular}

There was hyperidrosis pedum. The eruption was better in two weeks.

March 7, 1894: A prescription for treating seborrhea of the scalp was given the patient. No complaint was made of the feet.

May 10: On both soles, especially beneath the arches, there were pin-head to nail-head diameter vesicles and bullae, and note was made of the "same old trouble." A 1:60 potassium bichromate solution was ordered as a wash and a bidaily dressing:

R. Salicylic acid $\ldots \ldots \ldots \ldots \ldots \ldots \ldots \ldots \ldots \ldots$

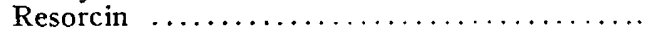

Magnesium carbonate $\ldots \ldots \ldots \ldots \ldots \ldots \ldots$

Olive oil........................... s.

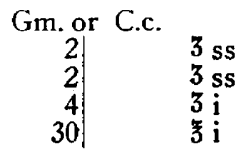

May 12: The old lesions were improved, and there were a few new ones.

The patient died within the following year. His was a typical case of phytosis, the summer type.

CASE 2.-Mrs. H. T., aged 29, first seen May 8, 1912, had pompholyx of the hands and feet, first noted in the spring of 1910, and again in the spring of the present year. On the palms, the fingers over the nail roots and on the toes, there were vesicles and erythematosquamous sequelae. On the soles and sides of the feet, there were bullae up to quarter of a dollar in size. Lesions were ordered broken up and boric acid and salicylic acid, one part each, zinc oxid (pulverized), eight parts, and talcum (pulverized) to make thirty parts was ordered kept on in white stockings and gloves.

The lesions began to dry, but new and painful flat bullae appeared, and new vesicles on the palms and fingers. Treatment, as for dermatitis or eczema. continued through prolonged dry, scaly and occasional vesicular periods, until August 28 when the affected areas showed but slight redness and scaling. All were healed, September 25 . 
May 7, 1921: The patient had had only one or two slight recurrences. At present, a vesicular to thick scaly itchy patch was present on the dorsum of one finger, and a small disk in the right submaxillary area. The latter soon healed, with simple treatment; the finger was resistant. Recurrence on the finger and a patch on one sole developed in September, and treatment was continued, still without recognition of the possibility of phyton infection, and certainly no treatment was given for this condition. The patient, who was hypersensitive, had plant dermatitis meanwhile, the last outbreak, on the forearms and hand, following gathering garden peas, in May, 1922, at which time the old dermatoses had long since disappeared.

CASE 3-A medical student, from the country, had had trouble with his feet, summer and winter, for more than a year. When first seen, the anterior half of the plantar epidermis of the left foot was undermined with pus, and there was the usual condition of the toes. The pus was rapidly eliminated with ointment of ammoniated mercury. A large callus beneath the anterior arch was gradually removed with salicylic acid and adhesive plaster. Dermatitis of the toes was reduced by the salicylic acid, calomel and talcum powder; but the use of salicylic acid alone for the whole foot and peeling the sole to infantile thinness really accomplished the cure. This case was fought from fall to spring and definitely cured. The feet were always damp, partly for the reason that he wore overshoes in bad weather and kept them on indoors.

CASE 4.-A college professor, seen recently in May, when the daily temperature was running above $80 \mathrm{~F}$, had had the condition for six summers, always affecting the external three toes of the right foot. It was never present in cold weather and had never been conveyed elsewhere, though the patient was most careless in handling the condition. Clinically, it was a perfect type of phytosis.

CASE 5.-This case is biographic of the disease as affecting the feet of a medical man with whom my relations were not exceeded by those of the Beaumont-St. Martin partnership. In 1918, he contracted the condition in quarters at a base hospital. This erythematosquamous dermatitis of lateral surfaces of the toes and interspaces, with fine fissuring of the latter, was attributed to the shower bath which was so strongly chlorinated that it irritated the eyes. The condition disappeared in cold weather.

In 1919, in Atlanta, there was recurrence with the advent of warm weather, vesicles now appearing about the toes. Diagnosis was made and careless stepping on a boarding house bath rug incriminated. All kinds of treatment and experiments were carried out; however, the salicylic and benzoic powders with talc were employed mostly, as well as occasional trials of most unusual procedures. The lesions disappeared by the time of frost, though the patient was rarely and briefly exposed to cold.

1920: The condition reappeared with the steadily warm days, it now involving also the anterior sole, from vesicles to small bullae. The slippers used in army were worn at home winter and summer. Summer socks, the same used even thus far in 1922, and an old pair of canvas shoes were worn during the hot weather, far into the summer of 1921. The disease had always appeared before these shoes were put on and even with new shoes. Slippers, socks and shoes got practically as much treatment as the feet.

1921: The toes had never been as uniformly affected as in the first year or two. Now the vesicles and deep bullae appeared as far back as the midsole, and two or three of the toes were showing small patches of vesicles dorsolaterally, especially near the nails. Vesicles were broken up and salicylic 
acid rubbed into all new areas or lesions. Gasoline was tried again in spite of its former bad effect on broken points, the socks being saturated with it and slippers or shoes put on. Later, ethyl chlorid freezing was practiced on all new outbreaks, and this season finished with the salicylic-sulphur-talc powder.

There was never hyperhidrosis, though there was a slight dampness of the socks winter and summer. Salicylic acid beneath adhesive plaster on a new patch caused a violent outbreak of vesicles beneath the plaster, peripherally to the salicylic acid application.

In April, 1922, there were a few days on which the temperature rose above $70 \mathrm{~F}$. My impression is that the same socks, some pairs of which had been worn for several summers, cotton or mercerized, bluish-gray, were resumed about this time. Promptly, a large pea-sized bulla appeared beneath the center of the right heel, and a dime-sized patch of vesicles developed on the inner side of the arch of the same foot. All lesions were broken and rubbed with salicylic acid, sometimes the salicylic-sulphur mixture, and for the following weeks the inside of the socks were heavily dusted with salicylic acid, four parts, precipitated sulphur, eight parts, and talcum (pulverized) to make thirty parts (mixed). There was no night treatment. The bullae never itched but they were painful. There was intense itching of the primarily vesicular patches, just as this year, long after there was nothing but a little redness and scaling. At the end of May, there had been no new lesions; the site of the bulla peeled off, and the little patch was pink, thinly desquamative and a bit itchy. Many days in May and June, 1922, the thermometer rose as high as from 82 to $85 \mathrm{~F}$. and the humidity was excessive. Use of the powder was continued and the left foot, thus far, has escaped and the right foot has shown no further lesions. ${ }^{2}$ The sulphur accentuates the peeling effect of the salicylic, much of the latter being synthetic and probably weak locally.

We are not prepared thus early to believe that the final chapter in this biography can be written. The same lot of socks, washed by a colored woman, is being worn; the shoes were new in the winter. There is a pair of oxfords of last summer yet to be tested, though personally I would be satisfied to let well enough alone. ${ }^{3}$

This patient will never step on any bath floor and has the greatest repugnance for shower baths-the cement floors of all of which we believe infected.

\section{SUMMARY AND CONCLUSIONS}

It is admitted that the term phytosis, an amputated extremity of more definitely descriptive names, is a makeshift. Epidermophytons appearing now in the minority in the tricophyton group, we cannot properly continue epidermophytosis as descriptive. If the term tinea, used since 1829 , means worm, it is as bad as many other misnomers. As it is impossible to make a laboratory diagnosis in every case, we must employ the loose term phytosis as a convenient designation.

2. The oxfords were later worn through a period of hot June weather, the temperature going as high as $93 \mathrm{~F}$. The powder was never omitted.

3. Post seu propter hoc, within forty-eight hours after putting on a pair of socks not worn since last summer a single pruritic vesicle appeared above the outer edge of right heel. Severe treatment produced terminal peeling on the fifth day. 
This disease group has few if any individually distinctive clinical features pointing to the type of fungus, even as the multitude of dermatitides venenatae may result from as many kinds of irritant as there are cases.

The disease is less contagious than infectious through an intermediary, as witness the frequency of unilateral cases and common sources of acquirement. Cases reported from practically every part of the world almost demonstrate pandemicity.

Commonest foci of infection are public baths, especially showers, and family contamination of rugs and floors and perhaps towels. Reinfection occurs from shoes, slippers and socks, even after laundering, or from laundry transference. The duration of viability of the fungi is from many months to an indefinite period, even in dry air. The majority of cases seems to indicate cold weather dormancy of organisms, even if the daily temperature reaches $60 \mathrm{~F}$.

There is some preponderance of evidence that the fungi live as saprophytes in the epidermis all winter, down-growth preventing their removal by normal desquamation, though reinfection from footwear also occurs.

We encounter the same difficulty in treatment that is common in other infective conditions, such as tuberculosis, syphilis and gonorrhea; and, as in the case of inaccessible cancer cells, destruction in vitro and in vivo is hence vastly different. It is essential that desquamation and exfoliation be constantly induced. Salicylic acid is the best agent for this purpose. Aside from the use of an ointment of ammoniated mercury in secondary pus cases, where it is specific, greasy applications, particularly in the daytime, are not only uncomfortable but ruinous to shoes. Dyes, from tincture of iodin through the list, are disagreeable and of doubtful efficacy.

All vesicles must be broken up, all bullae or lakes buttonholed, loose epidermis removed, soap and water freely used and salicylic acid, from 12 to 100 per cent., employed, as a peeling agent. Powders in socks, shoes and slippers have constituted regular treatment, whether salicylic-sulphur or talcum powder, this at present seeming the more effective, salicylic and sulphur equal parts, or, occasionally the salicylic alone. Salicylic acid beneath air tight adhesive is used for the removal of callosities.

If greasy applications are needed at night, Whitfield's ointment, or a salicylic-sulphur ointment or simple petrolatum is used. Ointment of ammoniated mercury seems ineffective against these fungi. Cases extending over years are reported.

631 Candler Building. 